\title{
Du côté de l'incipit de la Recherche : la genèse de la fiction selon Proust
}

\section{Maya Lavault}

\section{CpenEdition}

\section{Journals}

\section{Édition électronique}

URL : http://journals.openedition.org/genesis/1141

DOI : 10.4000/genesis. 1141

ISSN : 2268-1590

\section{Éditeur :}

Presses universitaires de Paris Sorbonne (PUPS), Société internationale de génétique artistique littéraire et scientifique (SIGALES)

\section{Édition imprimée}

Date de publication : 15 juin 2013

Pagination : $91-104$

ISBN : 978-2-84050-893-9

ISSN : $1167-5101$

\section{Référence électronique}

Maya Lavault, «Du côté de l'incipit de la Recherche : la genèse de la fiction selon Proust », Genesis [En ligne], 36 | 2013, mis en ligne le 08 juillet 2015, consulté le 19 avril 2019. URL : http:// journals.openedition.org/genesis/1141; DOI : 10.4000/genesis.1141 


\title{
Du côté de l'incipit de la Recherche: la genèse de la fiction selon Proust
}

\author{
Maya Lavault
}

$\mathrm{L}$

'évolution récente de la génétique textuelle autour de la position de la discipline au carrefour de l'herméneutique philologique et de la logique des possibles doit être mise en relation avec l'intérêt qu'ont porté ces dernières années un nombre croissant de critiques non seulement à la notion de " monde possible », mais aussi à la théorie des textes possibles ${ }^{1}$ - ce qui ne revient pas exactement au même ${ }^{2}$. Je pense en particulier à Daniel Ferrer ${ }^{3}$, qui s'est attaché à examiner les conditions de l'application de la notion de « monde possible » à la génétique, en partant du constat que la prolifération indicielle engendrée par le matériau génétique est à la fois enracinée dans la matérialité du document, à travers les signes tangibles de la création qu'il exhibe, et génératrice de " mondes possibles » vers lesquels pointent tous les signes contenus dans les avant-textes comme autant de scénarios hypothétiques dont l'existence, restée en suspens, n'est pas encore réalisée. S'appuyant sur l'hypothèse d'une possible analogie entre faits d'écriture et faits de lecture, sa réflexion pose les bases, mais suggère aussi les limites d'une appréhension des œuvres virtuelles esquissées par les manuscrits comme des mondes possibles que la critique génétique se donnerait pour tâche d'étudier, en comparant les propriétés des différents univers créés par les états successifs d'un même texte pour comprendre les transformations qui mènent de l'un à l'autre.

L'objectif de la réflexion proposée ici est de combiner génétique textuelle et théorie des textes possibles : dans la mesure où cette dernière a entrepris de forger une méthode d'analyse textuelle véritablement créatrice, qui entend confronter le texte « réel » à ce qu'il aurait pu être, elle partage avec la génétique la conscience du caractère relatif du texte, qui va de pair avec un intérêt pour ses virtualités étudiées en tant que telles, en même temps qu'une réflexion sur sa fabrique, sa cohérence interne et son fonctionnement. Il m'a semblé intéressant, dans la perspective d'une réflexion sur la genèse de l'œuvre qui tienne compte de l'émergence d'une conception de la fiction comme saisie des possibles qui la constituent ${ }^{4}$, de se pencher à nouveau sur les premières pages de la Recherche, déjà abondamment commentées par les généticiens ${ }^{5}$, mais beaucoup moins par les théoriciens des textes possibles. Dans la lignée de Michel Charles qui, dans Introduction à l'étude des textes, avait étudié la cohérence possible de ce qu'il désignait comme des " récits parasites ou récits fantômes 6 » qui « hantent » le début de la Recherche, je voudrais revenir sur les micro-récits, courtes saynètes ou

1. Théorie des textes possibles, dir. M. Escola, CRIN, n 57, 2012 (présentation de M. Escola, «Le chêne et le lierre. Critique et création », p. 7-18).

2. Voir La Théorie littéraire des mondes possibles, dir. F. Lavocat, Paris, CNRS Éditions, 2010.

3. D. Ferrer, « Le matériel et le virtuel : du paradigme indiciaire à la logique des mondes possibles », Pourquoi la critique génétique? Méthodes, théories, dir. M. Contat et D. Ferrer, Paris, CNRS Éditions, coll. «Textes et manuscrits », 1998, p. 11-30, et « Mondes possibles, mondes fictionnels, mondes construits et processus de genèse », Genesis, no 30, « Théorie : état des lieux », 2010, p. 109-130.

4. Je me permets de renvoyer à ma thèse de doctorat : " Des secrets à l'œuvre : formes et enjeux romanesques du secret dans À la recherche $d u$ temps perdu de Marcel Proust », Université Paris-Sorbonne, 2009, partie III (à paraître aux éd. Champion).

5. N. Mauriac Dyer fait le point sur le dossier génétique de l'ouverture de la Recherche dans «Découverte d'un inédit : du nouveau sur l'incipit d'À la recherche du temps perdu », Genesis, n 27, 2006, p. 141-157.

6. M. Charles, Introduction à l'étude des textes, Paris, Éditions du Seuil, coll. « Poétique », 1995, p. 169. 
« capsules7 » fictionnelles, qui jalonnent l'ouverture de l'œuvre pour en dégager une autre cohérence, à partir de l'étude des avant-textes. Il s'agira, non pas comme le fait Michel Charles, dans la perspective de ce qui s'apparente à une "génétique sans brouillons ${ }^{8}$ », de faire surgir, dans la synchronie de la lecture littéraire, d'autres récits possibles sous le texte lu, mais de montrer comment s'est progressivement construite, dans la diachronie de l'écriture, la cohérence des micro-récits de l'incipit, pour mettre en évidence à la fois le processus d'effacement et les traces de la persistance, au sein du texte publié, de scénarios abandonnés qui continuent de hanter le début du roman. Si la méthode adoptée est donc toute génétique, l'objectif poursuivi se veut résolument proche de celui de Michel Charles : il s'agit de retrouver les scénarios et les motifs qui ont servi à l'élaboration des premières pages de la Recherche pour montrer que ces possibles effacés au fil du processus de genèse continuent de « résonner » sous le texte publié.

L'étude des Cahiers 3, 5 et 1 permet de retracer la genèse de l'ouverture de «Combray », de la fin 1908 à 1909, au moment où Proust travaille au projet du Contre Sainte-Beuve. Ces pages, abondamment réécrites d'un cahier à l'autre, étaient à l'époque destinées au « récit d'une matinée 9 » censé introduire la « conversation avec Maman ». Le Cahier 3, premier des «Cahiers Sainte-Beuve », pose ainsi les jalons de l'expérience de désorientation spatio-temporelle du «dormeur éveillé ». L'énonciateur y est Marcel Proust lui-même : ayant pris l'habitude de ne dormir que le jour et de se coucher au matin, avant que «Maman » n'entre dans sa chambre lui apporter le journal, il guette les premières lueurs du jour qui, dans une semi-obscurité et un état de demi-sommeil, modifient les contours de sa chambre. Cette évocation amène une comparaison avec un malade obligé de coucher dans une chambre inconnue, qui sert de prétexte au développement d'un bref scénario imaginaire, opérant un glissement de la première personne - je du dormeur, nous de généralité - à la troisième personne d'une figure de voyageur anonyme située à mi-chemin entre cas clinique et personnage d'une fiction parallèle, quasi autonome :

J'étais couché depuis une heure environ. Le jour n'avait pas encore tracé attesstus [sic] des rideaux de ma fenêtre eette ligne blanehe qui dans l'obseurité de la ehambre dans la chambre łà à l'endroit où nous imaginions la commode $[\ldots]$. Parfois c'est une clarté, reflet d'une braise <qui a oublié de s'éteindre $>$ sur le cuivre d'un meuble $<$ d'une braise oubliée dans le feu éteint,> qui nous a trompé et que nous croyions déjà le jour audessus des rideaux de la fenêtre, moins triste que la raie de lumière qui, sous la p[orte] dans la chambre d'un hôtel inconnu, trompe le malade ; dressé sur son lit par une crise cruelle qui l'a réveillé, il voit cette lumière sous la porte et se dit c'est le jour, je n'entends pas encore de bruit, mais bientôt tout le monde va se lever, je n'aurai pas le on viendra me porter secours, je n'ai plus longtemps à attendre, et il compte les minutes. Bientôt la lumière sous la porte s'éteint et it retombe re rentre < dout rens l'obsurité. Il comprend, sa crise l'avait éveillé presque au moment où il venait // de s'endormir. Il est minuit. Dans l'hôtel inconnu on vie[nt] le garde de nuit don[t] il y a cinq minutes encore il aurait pu demander l'assistance vient d'éteindre le gaz, et il devra rester seul toute la nuit, à souffrir sans aide (Cahier 3, fos 1-2 ros10).

La focalisation interne sur le malade donne une autonomie au récit, en suscitant l'empathie du lecteur et en ménageant un bref suspense avant l'effet de « chute » final, tandis que l'utilisation du discours direct, qui reproduit les pensées du personnage à la première personne sans guillemets introductifs, établit une confusion entre le je du «dormeur éveillé » et celui de ce «malade imaginaire ». L'introduction, au sein de la rêverie du «dormeur éveillé », de cette micro-fiction centrée sur une figure autre que le je de l'énonciation, mais qui entre avec lui dans un jeu de partage des référents, a toute son importance. C'est en effet le seul élément de l'incipit qui soit en place dès les toutes premières versions proposées dans le Cahier 3 : les réécritures suivantes n'en modifieront pas la place,

7. J'emprunte cette expression à J. Dubois («Petits éléments de fiction théorique », dans Essai et fiction, BIP, n 42, 2012, p. 109-112).

8. Selon 1'expression de M. Escola dans « Le rêve et la formule : de l'éclair de génie au bon à tirer, l'analyse génétique », [en ligne], <www. fabula.org/revue/cr/80.php > (compte rendu de P.-M. de Biasi, La Génétique des textes, Paris, Nathan, coll. « $128 », 2000)$.

9. Corr., t. VIII, p. 320.

10. Transcription de B. Brun, dans « Le dormeur éveillé, genèse d'un roman de la mémoire », Cahiers Marcel Proust 11, Études proustiennes IV, Paris, Gallimard, 1982, p. 244-245. 
mais seulement la rédaction, dans le sens à la fois d'une plus grande autonomie de la «capsule » fictionnelle et d'un raccord plus souple avec la trame narrative principale. Ainsi, la version publiée en 1913, fixée à quelques détails près dès le Cahier 1 (fos 71-70 vos), développe par le truchement du discours indirect libre à la troisième personne les pensées intimes du malade, tandis que la chute, par le rappel de l'heure et l'utilisation de la tournure impersonnelle, permet le raccord avec la situation du « dormeur éveillé11 ».

Bien plus loin dans le Cahier 3, au folio 18 ro, l'organisation de ces premiers fragments constituant l'incipit est bouleversée par une invention capitale, qui donne aux premières pages de l'œuvre leur dynamique et au système énonciatif qu'elles mettent en place sa singularité : il s'agit de l'opposition entre l'époque révolue où le je dormait la nuit - à laquelle renvoie la désormais célèbre formule liminaire «Longtemps je me suis couché de bonne heure », introduite en 1911 sur la première dactylographie corrigée du «Temps perdu 12 » - et l'époque actuelle, qui correspond au présent de l'énonciation, où il ne dort plus que le jour. À la suite du Cahier 3, le Cahier 5 exploite cette opposition structurelle en introduisant une réflexion sur le sommeil d'autrefois, propice aux rêves :

Jusque vers l'âge de vingt ans, je dormis la nuit. Mon sommeil n'était qu'une $<$. Une $>$ sorte de eommunion avee $<$ participation à > l'obscurité de la chambre et à la vie inconsciente de ses [...] cloisons et de ses meubles $<$ tel était mon sommeil.> [...] D'ailleurs j'avais mes rêves. Je resstseitais à une vie où je n'avais j'éprouvais en dormant les sensations des années // pendant lesquels j'éprouvais ces sensations, ces idées bizarres d'un autre temps que nous ne pensions plus pouvoir jamais ressentir. [...] C'est ainsi que souvent mes réveils étaient causés par l'épouvante de m'être aperçu que mon onele <notre curé> arrivait à pas de loup derrière moi pour me tirer par mes boucles, ee qui avait été l'épouvante te eauchemard $[\mathrm{sic}]<\mathrm{l}$ 'effroi $>$ de mon enfanee aete par lequel Havait empoisonné monenfanee <ce qui avait été la terreur et le supplice de mon enfance. $><$ Je voulais me sauver, il les tirait, j'essayais de me détourner, je ne pouvais pas, je m'éveillais.> Or il y avait bien des années que je n'avais plus de boucles, plus de <mon> grand oncle [...] (Cahier 5, fos $113-112 \mathrm{vos}^{\mathrm{os}}$.
L'épisode des boucles tirées prend dans les Cahiers 5 et 1 une importance majeure : Proust le retravaille successivement, le développant parfois très longuement, pour introduire une réflexion sur les «terreurs », « effrois » et autres « supplices » d'une enfance à jamais révolue, que son narrateur ne peut se remémorer que dans le sommeil, par le truchement du rêve. Dans les versions successives de cet épisode, l'homme qui tirait les boucles est tour à tour le curé de Combray, l'oncle et le grandoncle du protagoniste (Cahier 5, fos 113, 112, 110 vos : voir fig. 1 et fig. 3 ; Cahier 1 , fo 69 vo), puis son grandpère 13 (Cahier 1 , fo 65 ro) ; dans la version définitive, qui est plus condensée, c'est le grand-oncle qui joue ce rôle14. Ce souvenir d'enfance retrouvé dans le sommeil s'inscrit dans un espace de «mémoire indécise15 » à la fois onirique et autobiographique, qui pourrait bien

11. «J'appuyais tendrement mes joues contre les belles joues de l'oreiller [...]. Je frottais une allumette pour regarder ma montre. Bientôt minuit. C'est l'instant où le malade, qui a été obligé de partir en voyage et a dû coucher dans un hôtel inconnu, réveillé par une crise, se réjouit en apercevant sous la porte une raie de jour. Quel bonheur, c'est déjà le matin ! Dans un moment les domestiques seront levés, il pourra sonner, on viendra lui porter secours. L'espérance d'être soulagé lui donne du courage pour souffrir. Justement il a cru entendre des pas ; les pas se rapprochent, puis s'éloignent. Et la raie de jour qui était sous sa porte a disparu. C'est minuit ; on vient d'éteindre le gaz ; le dernier domestique est parti et il faudra rester toute la nuit à souffrir sans remède » $(\mathrm{I}, \mathrm{p} .4)$.

12. C'est le titre donné par Proust à la première partie de son roman en 1911 : voir N. Mauriac Dyer, art. cité, p. 141-142.

13. Ces figures masculines occupent toutes dans le système des personnages du roman une place de «précepteur » à la fois bienveillant et persécuteur - risquons l'hypothèse que c'est peut-être la proximité phonétique des termes précepteur et persécuteur qui motive l'emploi du mot précepteur dans le Cahier 5 où le terme est d'abord biffé, puis réintroduit au folio $112 \mathrm{vo}$, traduisant ainsi sans doute l'ambivalence liée à cette fonction. Rappelons que c'est la « férocité inconsciente » du père et du grand-père qui est à l'origine du « supplice » du coucher de l'enfant (I, p. 27) : nous verrons plus loin le lien que cet épisode entretient justement avec le « drame du coucher ».

14. « Ou bien en dormant j'avais rejoint sans effort un âge à jamais révolu de ma vie primitive, retrouvé telle de mes terreurs enfantines comme celle que mon grand-oncle me tirât par mes boucles et qu'avait dissipée le jour - date pour moi d'une ère nouvelle - où on les avait coupées. J'avais oublié cet événement pendant mon sommeil, j'en retrouvais le souvenir aussitôt que j'avais réussi à m'éveiller pour échapper aux mains de mon grand-oncle, mais par mesure de précaution j'entourais complètement ma tête de mon oreiller avant de retourner dans le monde des rêves » (I, p. 4). 15. Selon la belle expression de M. Charles (op. cit., p. 203). 
trouver à s'insérer dans la trame de «Combray » : il possède un « air de famille » évident, quoique rarement décelé, avec l'épisode de l'adieu aux aubépines, où il est justement question des boucles du héros enfant. Voici la première rédaction de l'épisode dans le Cahier 29, daté de 1909-1910 :

Les années où j'étais je fus obligé de quitter Combray après les fêtes <lendemain > de la Pentecôte, je trouvais mes parents barbares de me faire quitter mes chères aubépines. J'allais teur dire adieu, je les pressais sur mon eeur, arrachant ma veste à la grande colère de Maman. Ce n'est pas vous qui voudriez me faire de la peine, mes pauvres petites aubépines teur disais-je en pletrant. Mon gr oncle pour me faire plaisir coupait de grandes branches qu'il nous donnait en partant <en nous disant adieu sur le pas de la porte*>, mais Maman n'avait rien de plus pressé que de les jeter ou de les laisser dans la voi < de faire > // semblant de les oublier dans la voiture, disant que rien n'est plus ennuyeux que des fleurs en voyage. Fe savais aumoment où nous Aussi sachant que je ne les verrais plus le matin du départ je me sortais de bonne heure et j'allais leur dire adieu. Je les pressais sur mon cœur, ce qui me faisait à la grande colère de Maman, revenir avec une veste toute arrachée. « Ce n'est pas vous qui chercheriez à me faire de la peine, à me forcer à quitter ce que j' aime < <etri disais-je> ». Jamais, ce n'est pas vous qui me feriez pletrer mes pauvres petites aubépines, leur disais-je, jamais vous ne m'avez fait de chagrin, vous ", leur disais-je en pleurant (Cahier 29, fos 71-72 vos16).

C'est bien une manière de scène de sadisme, traitée sur le mode de la distanciation ironique, qui se dessine ici : le rôle de «bourreau » est attribué aux parents «barbares » qui obligent le héros à quitter ses « chères » aubépines, et plus particulièrement à la mère, qui s'empresse de se débarrasser des fleurs importunes. L'oncle y occupe un rôle inverse de celui qu'il tient dans l'épisode des boucles tirées : c'est lui qui coupe les branches d'aubépines pour faire plaisir à son jeune neveu. La parenté des deux épisodes apparaît plus clairement encore à la lecture de la première version connue de la scène des aubépines, centrée sur le frère du protagoniste, Robert, en proie au désespoir de quitter un chevreau ${ }^{17}$. L'oncle a emmené Robert se faire photographier à Évreux après lui avoir fait friser les cheveux : avec ses « cheveux noirs bouffants » et ses « grands nœuds », sa « petite robe » et sa « jupe de dentelle », le jeune enfant est comparé à une " infante de Velasquez », qui prend l'attitude d'une «princesse de tragédie pompeuse et désespérée », comparaison qui amène la référence à Phèdre; accusant ses « persécuteurs » de vouloir l'arracher à un chevreau pour lui faire prendre le train en tenant des propos raciniens qui annoncent ceux du jeune héros de la Recherche, il finit par arracher les nœuds de ses cheveux, puis par s'asseoir sur la voie de chemin de fer, contraignant ses parents à l'arracher aux rails.

En effet, l'épisode « Robert et le chevreau » peut être mis en relation avec un fragment du Cahier 6 qui y fait directement allusion, en marge de l'évocation de la tristesse du héros lorsque sa mère quittait Combray :

Moi je ne $<$ ne $>$ voyais au contraire jamais sans tristesse les clochers de Chartres, car souvent c'est jusqu'à Chartres que nous accompagnions Maman quand elle quittait Combray avant nous. Et je voyais // et la forme horrible <inéluctable> des < deux > clochers m'apparaissait aussi terrible que la gare. J'allais vers eux comme vers le moment où il faudrait dire adieu à Maman, sentir mon cœur s'ébranler dans ma poitrine, se détacher de moi pour la suivre et revenir seuls [sic]. Je me souviens d'un jour particulièrement triste où Maman emmenait mon frère dans la voiture devant nous conduire de Combray à Chartres et c'était bien loin. On avait faire $[\mathrm{sic}]$ photographier mon frère le matin avant qu'il partît (Cahier 6, fos 69-68 vos18).

16. Transcription de B. Brun, dans « Brouillons des aubépines », Cahiers Marcel Proust 12, Études proustiennes V, Paris, Gallimard, 1984, p. 232; transcription revue.

17. L'épisode est désigné sous le titre «Robert et le chevreau » dans le Carnet 1, qui contient la liste des « Pages écrites » que Proust destinait au roman auquel il travaillait au premier semestre 1908, avant de se lancer dans le projet du Contre Sainte-Beuve (Carnets, éd. F. Callu et A. Compagnon, Paris, Gallimard, 2002, p. 43, n. 79). Certaines de ces pages correspondent aux soixante-quinze feuillets manuscrits dont subsistent des fragments reproduits dans le volume Contre Sainte-Beuve édité par B. de Fallois en 1954 : c'est le cas de l'épisode « Robert et le chevreau » (Contre Sainte-Beuve, Paris, Gallimard, coll. « Folio Essais », 2002, p. 286-290).

18. Transcription d'A. Wada, "L'évolution de "Combray" depuis l'automne 1909 », thèse de doctorat, Université de Paris IV, 1986, t. II, p. 13-14 ; transcription revue par J. André en vue de l'édition du Cahier 6 dans la collection des Cahiers 1 à 75 (BnF-Brepols). B. de Fallois raccorde les deux fragments au sein de l'épisode « Robert et le chevreau » dans son édition du Contre Sainte-Beuve (op. cit., p. 285). 

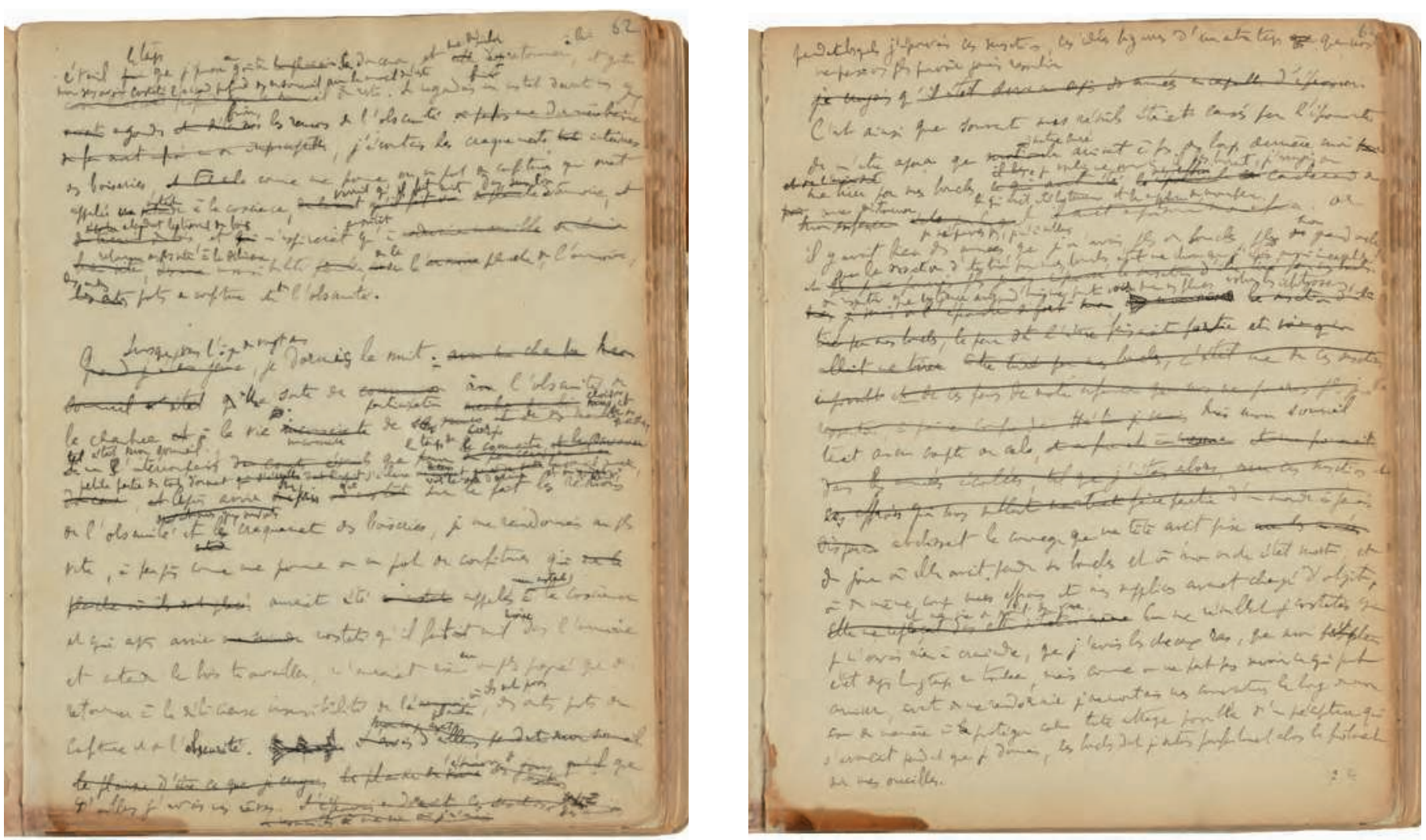

Fig. 1 : Cahier 5, fos 113 et 112 vos

(BnF, NAF 16645)

La scène du chevreau, centrée sur le petit frère, s'inscrit donc dans le contexte d'une autre séparation, celle du protagoniste avec sa mère, comme si ce motif essentiel était masqué par une autre séparation qui vient en redoubler la violence, mais de manière détournée, par déplacement du héros à Robert et de « Maman »au chevreau. C'est ce que dit explicitement la fin de l'épisode " Robert et le chevreau », où la fureur du héros rejoue avec un mimétisme confondant la «tragédie » vécue par le petit frère :

Une ou deux fois sur la route, une sorte de fureur m'envahit, je me considérais comme persécuté par elle et mon père, qui m'empêchait de partir avec elle, j' aurais voulu me venger en lui faisant manquer le train, en l'empêchant de partir, en mettant le feu à la maison ${ }^{19}$.

Dans les deux versions de l'épisode - le chevreau et les aubépines -, les parents tiennent le rôle de «persécuteurs », rôle attribué dans le souvenir des boucles tirées de l'incipit au grand-oncle/grand-père/ oncle/curé qui, dans « Robert et le chevreau », joue sous les traits de l'oncle un rôle au contraire très positif. Tout se passe donc comme si l'ambivalence de cette fonction générique de précepteur avait été résolue par une distribution quasi axiologique des rôles : aux parents est attribué le rôle de « bourreaux », à « l'oncle » celui de conciliateur. Le souvenir des boucles tirées introduit au contraire une autre partition : c'est l'oncle à l'identité instable qui y joue le rôle de persécuteur, annonçant ainsi le motif essentiel du «drame du coucher » où l'ambivalence de la fonction parentale, à travers les figures du père, du grand-père et de la mère du héros, est pleinement exploitée. Le micro-récit centré sur le souvenir des boucles peut en ce sens apparaître comme une préfiguration du « supplice » du coucher, dont il propose une version atténuée, presque expurgée, et détournée : ainsi pourrait-on expliquer que la rédaction définitive de l'épisode ait atténué l'idée de "supplice », réservée au récit du coucher, en adoptant le terme plus neutre de « terreur».

19. Fin de l'épisode «Robert et le chevreau » : voir CSB, op. cit., p. 291. 
Un autre fragment du Cahier 6 relie explicitement la séparation subie par le protagoniste à chaque départ de Combray aux retours de promenade du côté de Guermantes qui le privaient du baiser maternel20 :

C'est comme cela que je l'avais vu [le clocher de Chartres] que $[s i c]$ je rentrais des promenades du côté de Guermantes et que tu ne devais pas venir me dire bonsoir dans mon lit, comme cela que je le voyais quand nous t'avions mise en chemin de fer et que je sentais que c'était dans une ville où tu ne serais plus qu'il allait falloir vivre (Cahier 6, fo 7 ro21).

Mettre ce fragment en relation avec l'épisode des boucles tirées permet alors de lire sous le récit de ce souvenir enfoui d'une « terreur enfantine » un « texte fantôme » qui dit l'angoisse de la séparation avec la mère - séparation désignée en creux par le motif symbolique de la coupe (des cheveux, des aubépines), et plus explicitement par la violence de la réaction du protagoniste face à ses «persécuteurs ». La violence de l'épisode est, on l'a vu, très atténuée dans le texte publié, et son lien avec la scène des aubépines - qui n'est décelable qu'à la lecture de l'avanttexte « Robert et le chevreau »-totalement occulté :

[...] le matin du départ, comme on m'avait fait friser pour être photographié, coiffer avec précaution un chapeau que je n'avais encore jamais mis et revêtir une douillette de velours, après m'avoir cherché partout, ma mère me trouva en larmes dans le petit raidillon, contigu à Tansonville, en train de dire adieu aux aubépines, entourant de mes bras les branches piquantes, et, comme une princesse de tragédie à qui pèseraient ces vains ornements, ingrat envers l'importune main qui en formant tous ces nœuds avait pris soin sur mon front d'assembler mes cheveux, foulant aux pieds mes papillotes arrachées et mon chapeau neuf (I, p. 143).

L'épisode condense les éléments essentiels du souvenir des boucles tirées en les inversant : le héros arrache volontairement les boucles soigneusement agencées par le coiffeur, et entoure de ses bras, non pas ses cheveux, comme dans le souvenir des boucles, mais les aubépines et leurs "branches piquantes ». Derrière la dramatisation ironique qu'opèrent la comparaison avec « une princesse de tragédie » et la citation de Phèdre, ce qui est donné à lire en filigrane, c'est bien une scène de supplice : un supplice causé par la séparation dont la violence est détournée sur les « vains ornements » dont est affublé le héros. Les deux épisodes entretiennent donc un lien de parenté profond, mais enfoui : ils disent tous deux, par déplacement, le supplice de la séparation avec la mère, motif effacé au fil des réécritures, mais qui continue de hanter, comme un scénario fantôme, l'incipit du roman.

L'hypothèse demande à être confortée par l'analyse des autres micro-récits introduits dans les versions successives de l'incipit. Le Cahier 1, reprenant à la suite des Cahiers 3 et 5 l'ouverture de l'œuvre, introduit en amont de l'épisode du voyageur malade une première ébauche fictionnelle, absente des versions précédentes, centrée sur une autre figure de voyageur :

Je rallumais < la lampe>, je regardais l'heure, il n'était pas encore minuit, j'entendais le sifflement tointain $<$ plus ou moins éloigné> des trains qui décrit l'étendue de la campagne déserte, où sans doute au clair de lune se hâtait hâte le voyageur qui va rejoindre la prochaine gare, avee ette gâtté <exeitation> < gâtté> que donne le silenee de la nuit, la // nouveauté du voyage ou le plaisir du retour, quelquefois le bon dîner que nos hôtes nous ont fait faire, avant de nous faire reeonduire à la gare proehaine.

égayé par une de ees belles ntuits < route[s]> sur une route $<$ par une de ces belles nuits pavées de clair de lune $>$ que $<$ sont en train de $>$ grave $<$ r $>$ dans son souvenir la nouveauté $<$ 'excitation $>$ du voyage, du elair de lune $<$ du silence $>$, te plaisir du retour, te bon dîner qu'il a fait chez des hôtes à quil il a promis de revenir le plaisir goûté avec les amis qu'il vient de quitter, le plaisir du retour (Cahier 1, fos 71-70 vos, voir fig. 2a).

20. Selon la liste des « Pages écrites » qui figure dans le Carnet 1 , fo 7 vo, cet épisode du départ maternel devait suivre immédiatement dans le projet romanesque ébauché celui du chevreau, et précéder la distinction des deux côtés, ainsi que ce qui apparaît comme le canevas d'un « drame du coucher » : «Robert et le chevreau, Maman part en voyage. / Le côté de Villebon et le côté de Méséglise. / Le vice interpré sceau et ouverture du visage. La déception qu'est une possession, embrasser le visage. / Ma gd mère au jardin, le dîner de M. de Bretteville, je monte, le visage de Maman alors et depuis dans mes rêves, je ne peux m'endormir, concessions etc. [...] » (Carnets, éd. citée, p. 43, transcription revue).

21. Voir CS, I, p. 663 («Esquisse VI. $2 »$ ); transcription revue par J. André. B. de Fallois raccorde également ce fragment à l'épisode « Robert et le chevreau » (CSB, op. cit., p. 292). 


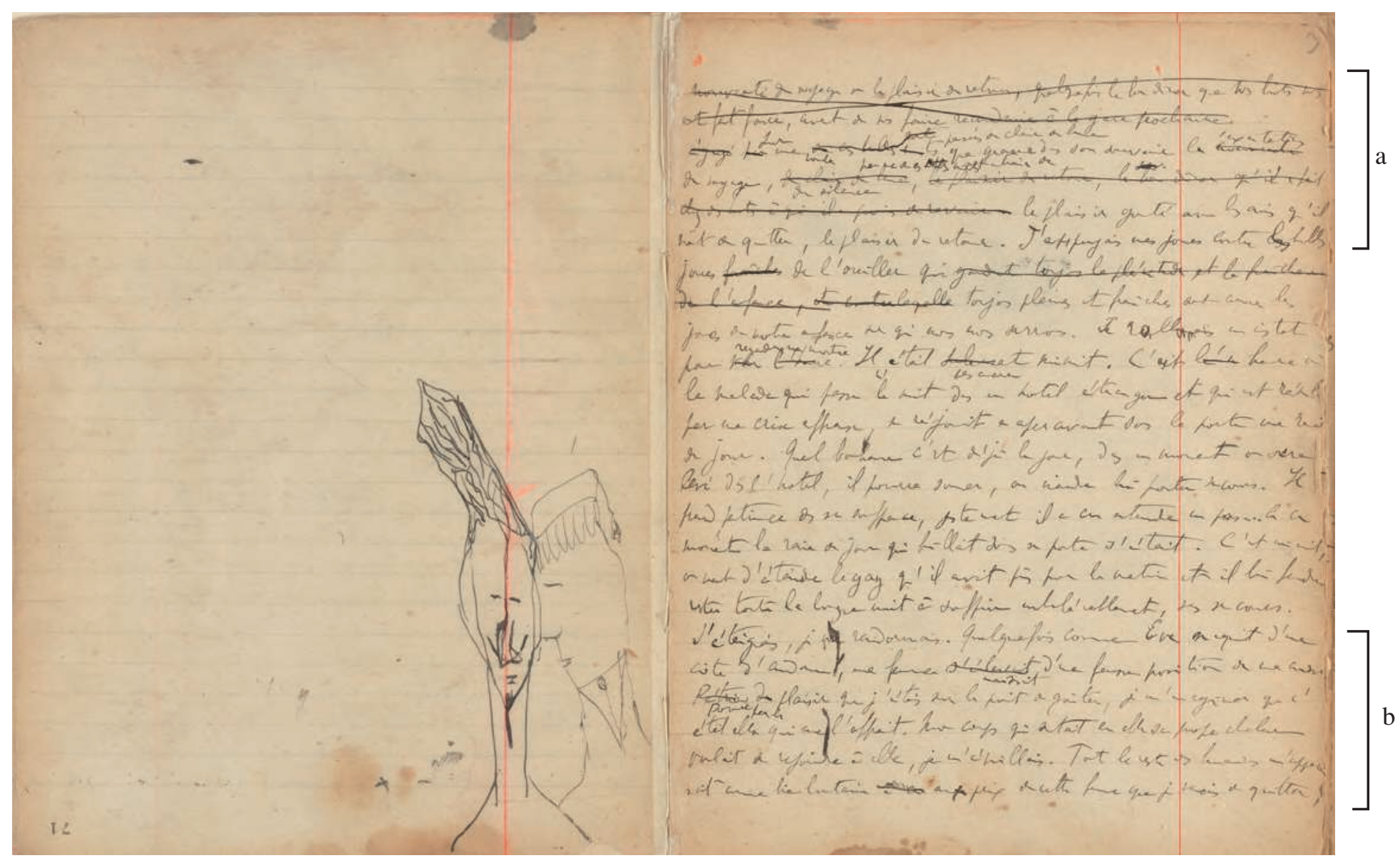

Fig. 2 : Cahier 1 , fos $71 \mathrm{r}^{\mathrm{o}}-70 \mathrm{v}^{\mathrm{o}}$

(BnF, NAF 16641)

Cette figure anonyme de voyageur est dotée d'attributs biographiques et psychologiques certes embryonnaires, mais suffisamment précis pour lui conférer une certaine autonomie, comme dans le cas du malade obligé de coucher dans une chambre inconnue : ainsi, ce premier scénario peut se lire comme l'expression furtive de la douceur d'un retour au foyer qui préluderait à l'angoisse que met en scène le second micro-récit centré sur le voyageur malade. L'enchaînement de ces deux saynètes contribue à associer dès l'incipit le voyage en train avec un sentiment d'angoisse22, et plus précisément avec "l'effroi de coucher dans une chambre inconnue », selon les termes employés par Proust dans un ensemble thématique développé dans le Cahier 26 (fos 57 à 60) autour de l'évocation de la chambre angoissante que seule permet d'apprivoiser l'habitude.
Ce développement fut d'abord intégré en octobrenovembre 1909 à la mise au net du texte de «Combray » dans le Cahier 9, puis supprimé sur la dactylographie pour être finalement déplacé au sein du récit de la première nuit du héros au Grand-Hôtel de Cricquebec, futur Balbec, dans le Cahier 70 (fos 43-45 ros23). Dans le texte définitif, l'évocation des chambres tournoyantes au début de «Combray » garde la trace ténue de ce développement sur l'habitude (I, p. 8), mais le motif de « l'effroi » en a été effacé : là encore, tout se passe comme si la version donnée par le texte publié avait été expurgée de l'idée

22. Voir F. Leriche, «Attention, un train peut en cacher un autre », Surprises de la Recherche, Textuel, n 45, 2004, p. 91.

23. Voir Cahier 26, éd. F. Leriche, A. Wada et H. Yuzawa, Turnhout, BnF/ Brepols, 2010, vol. II, n. 1 du folio 61 ro (p. 175-176). 
lancinante de «supplice », qui a été déplacée plus loin, lors de la première nuit du héros à Balbec dans les Jeunes filles (II, p. 30-32). Juste avant ce passage, le récit du voyage vers Balbec met en scène la tension qui déchire le héros : il est pris entre son désir de voyage suscité depuis longtemps par la lecture de l'indicateur des chemins de fer et l'angoisse de coucher dans une chambre inconnue, encore redoublée par la pensée que sa mère ne sera pas du voyage (II, p. 6).

Le train apparaît dans cet épisode à la fois comme un refuge désirable, en ce que la nuit qu'y passe le héros repousse l'échéance de la «cruelle première nuit où [il] entrer[a] dans une demeure nouvelle et accepter[a] d'y vivre » (II, p. 8) et comme l'instrument de la séparation avec la mère. Le récit du départ à Balbec reprend en effet l'épisode primitif de « Robert et le chevreau » suivi de "Maman part en voyage », qui est donc doublement réinvesti dans la Recherche : tandis que la violence du sentiment causé par la séparation - essentiellement assumée par Robert dans la scène du chevreau - s'exprime dans la scène de l'adieu aux aubépines, l'analyse à la fois dramatisée et ironique de ce sentiment est livrée dans la scène du départ pour Balbec. Notons enfin que le héros, au moment de ce voyage, est un malade, convalescent tout juste sorti de son amour pour Gilberte, et surtout sujet à des «crises de suffocation » qui ruinent sa santé, comme le voyageur du second micro-récit.

Revenons maintenant à la version de l'incipit donnée dans le Cahier 1 : pour la première fois dans le processus de genèse, les quatre micro-récits qui constitueront l'ouverture de Du côté de chez Swann en 1913, juste avant l'évocation tournoyante des chambres, sont en place et dessinent une cohérence qui donne une dynamique nouvelle à ce début d'œuvre. Tandis que les deux premiers sont centrés sur une figure de voyageur au sein de scénarios imaginés par le protagoniste qui ne s'est pas encore endormi - ils sont étroitement liés par la mention de l'heure : «il n'était pas encore minuit » répète le narrateur (fos 71-70 vos) -, le suivant introduit une rêverie érotique provoquée par l'engourdissement du corps dans le tout premier stade du sommeil :

Quelquefois comme Ève naquit d'une côte d'Adam, une femme s'élevait <naissait> d'une fausse position de ma cuisse. Pétrie du <Formée par le $>$ plaisir que j'étais sur le point de goûter, je m'imaginais que c'était elle qui me l'offrait. Mon corps qui sentait en elle sa propre chaleur voulait se rejoindre à elle, je m'éveillais. Tout le reste des humains m'apparaissait comme bien lointain à ế au prix de cette femme que je venais de quitter, // avee qui j'avais connu tant de plaisirs ; e'était la plus réeente la plus d et je l'avais vue de eette manière j' avais la tête $<$ joue encore $>$ chaude encore de ces [sic] baisers, le corps courbaturé par le poids de sa taille. Puis Bientôt $<$ Peu à peu $>$ je java son souvenir se dissipait s'évanouissait; et bientôt j'avais oublié la fille de mon rêve <maintenant*> aussi vite que si c'eût été une amante véritable (Cahier 1, fos 70-69 vos, voir fig. 2b).

Cette rêverie érotique avait été introduite, dans une rédaction très proche de celle-ci, au sein d'une addition au folio 111 ro du Cahier 5, qui développait le souvenir des boucles tirées, comme si dans le processus de genèse, le travail autour de l'épisode des boucles avait suscité, comme une excroissance, l'écriture du songe sensuel (fig. 3). Dans le Cahier 1, l'évocation érotique précède immédiatement le souvenir des boucles tirées, alors que dans la version publiée c'est elle qui clôt la série des premiers micro-récits du «dormeur éveillé », en venant se placer juste après le souvenir des boucles tirées, le réveil marquant alors simultanément la fin du songe et la projection de la femme fantasmée dans la réalité du protagoniste qui se rêve en « voyageur ${ }^{24}$ » idéaliste.

Après le développement du souvenir des boucles tirées, le Cahier 1 contient le récit d'une scène de masturbation juvénile dans le cabinet « qui était en haut dur ehâteatret $<$ de notre maison à Combray $>$ où des colliers de graines d'iris étaient suspendus » (fo 68 vo) (fig. 4) : il s'agit de la réécriture d'un fragment déjà développé, mais interrompu, au folio 109 du Cahier 5, autour du thème des sensations ressurgies d'un âge lointain de l'existence par le truchement des rêves nés d'un premier sommeil. Les deux scènes - la rêverie érotique et la scène de masturbation juvénile - $\mathrm{y}$ apparaissent thématiquement liées au récit des boucles tirées : « Le plus

24. «Si, comme il arrivait quelquefois, elle avait les traits d'une femme que j'avais connue dans la vie, j'allais me donner tout entier à ce but : la retrouver, comme ceux qui partent en voyage pour voir de leurs yeux une cité désirée et s'imaginent qu'on peut goûter dans une réalité le charme du songe » (I, p. 5$)$. 


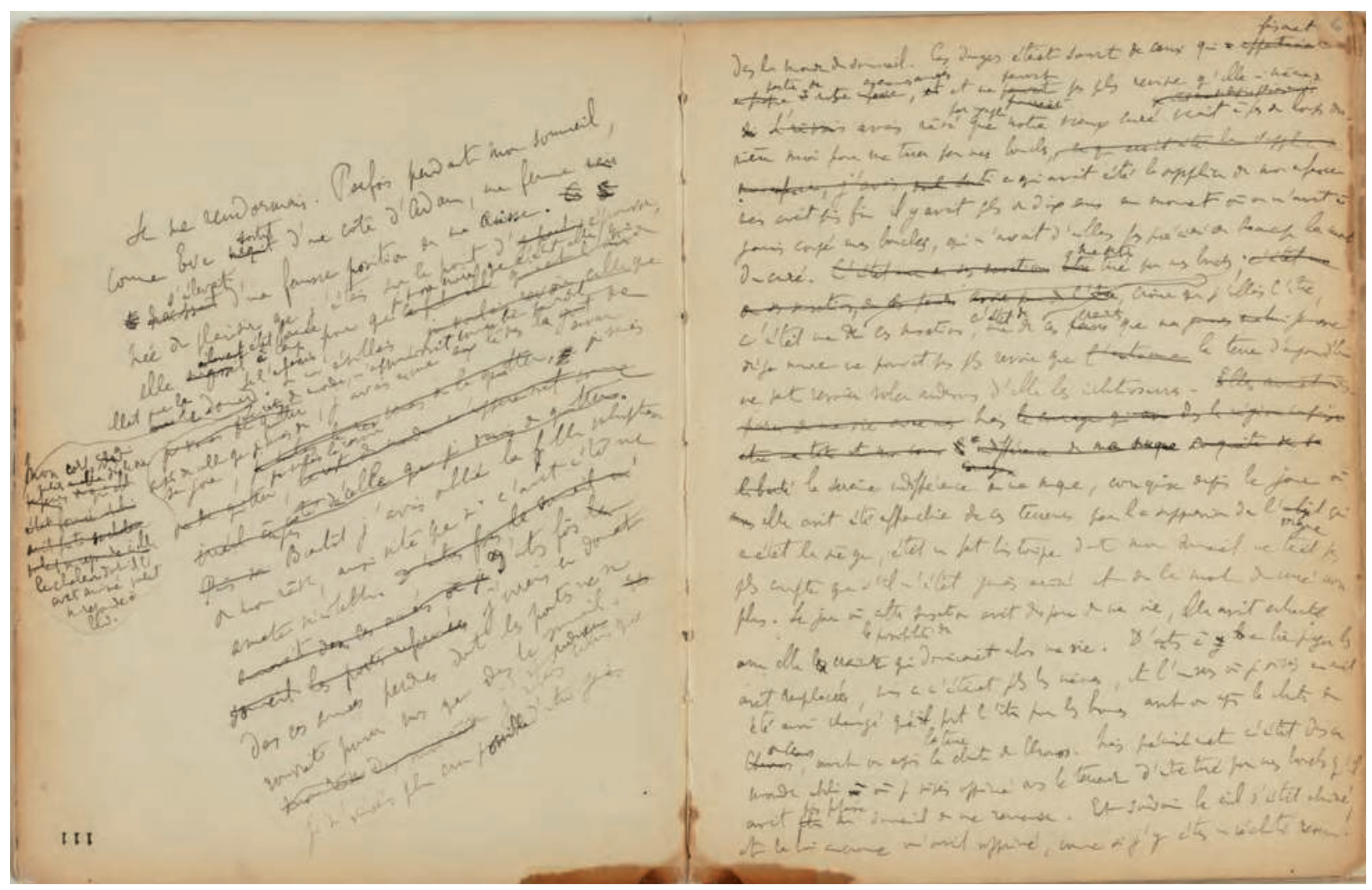

Fig. 3 : Cahier 5, fos 111 ro-110 vo (BnF, NAF 16645)

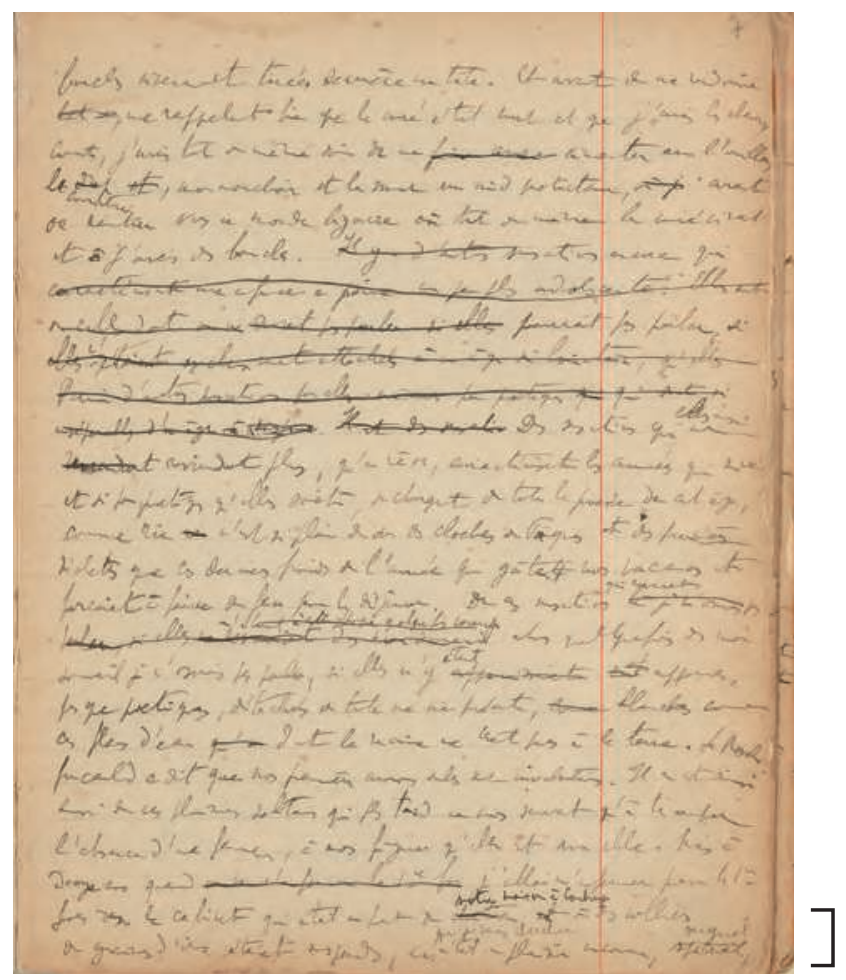

Fig. 4 : Cahier 1, fo 68 vo (BnF, NAF 16641) 
souvent je dormais / Si parfois je reprenais <aisément> en dormant cet âge inaeessible <enfui> <irretrouvable> où l'on a des botreles <craintes> et des désirs <plaisirs $>$ aujourd'hui inexplicables, le plus souvent je dormais sans rêves [...] » (Cahier 1, fo $\left.64 \mathrm{v}^{\mathrm{o}}\right)$. Est ensuite longuement retravaillé l'épisode des boucles : cette insistance fait de ce souvenir enfoui d'une «terreur » primitive un moment essentiel de l'ouverture de l'œuvre, dont l'importance a été amoindrie dans le texte publié, où il se trouve, sous une forme condensée, intercalé entre le micro-récit centré sur le voyageur malade et la rêverie érotique 25 .

Plus loin dans le Cahier 1, apparaît un autre récit de souvenir, longuement développé à la suite de l'évocation des chambres : il s'agit du séjour du protagoniste au château de Réveillon. L'évocation des retours de promenade à Réveillon est intéressante à plus d'un titre : d'abord parce qu'elle est discrètement reliée à la scène de masturbation juvénile qui, dans sa première rédaction, prenait place « en haut du château » (le terme a été biffé au folio 68 vo pour être remplacé par « en haut de notre maison à Combray ») ; ensuite, parce qu'elle mêle des éléments qui, dans le texte publié, seront distribués dans le récit des retours de promenade à Combray et dans l'épisode de la lanterne magique26. En voici le dernier développement, qui clôt une série de fragments où l'épisode est successivement retravaillé (fig. 5) :

nous sortions du village <; > sur une moitié des champs le couchant s'était éteint ; sur l'autre, la lune était déjà allumée ; nous ne reneontri nous traversions je traversais la grande rue du village où çà et là une boutique éclairée de l'intérieur par eomme un aquarium et remplie par la lumière onctueuse et pailletée de la lampe nous montrait sous sa paroi de verre des personnages prolongés, qui < prolongés de leur ombre> déplaçaient avec lenteur $<$ leurs ombres noires $>$ dans la liqueur d'or et nous présentaient les seènes l'apparenee un table des seènes variées dans une scène usuelle et fantastique le secret éclatant $<$ et surpris> de leur vie ; puis nous ne rencontrions plus que quelques le triangle irrégulier bleuâtre et mouvant des moutons qui rentraient, puis j'arrivais dans l'immensité les champs dont ; sur une moitié le couchant s'était éteint ; sur l'autre la lune était déjà allumée, bientôt le clair de lune les remplissait tout entières. Je m'avançais < dans leur étendue ta plaine> comme une barque qui a une longue navigation à aeeomplir qui $<$ rapidement> accomplit < de nuit> sa navigation de nuif $<$ solitaire > ; ...] j'avais < taissé > derrière moi une traversé, puis laissé derrière moi une étendue enchantée. Qutelquefois ła dame du château m'aeeomp Quelquefois la dame du château m'accompagnait $[\ldots]$ dans < le mystère $>$ d'une vallée profonde tapissée par le clair de lune [...] nous nous arrêtions un instant ma compagne et moi devant le mystère <attsetuil de> < avant de descendre dans ce> calice d'opâle [sic] [...] (Cahier 1, fos 59 ro-58 vo ; transcription simplifiée).

Les éléments de cette description d'un paysage de rase campagne semi-imaginaire, éclairé par la lune et dominé par un château fantasmatique dont le nom semblait «ne devoir se trouver que sur une carte du Rêve » (fo 58 vo), semblent avoir été réinvestis dans le récit de la chevauchée fantastique de l'affreux Golo se dirigeant vers le château de Geneviève de Brabant sous les irisations de la lanterne magique : ainsi, la « lumière onctueuse et pailletée de la lampe » qui montre sous « sa paroi de verre » des personnages « dans une scène usuelle et fantastique » évoque le dispositif de la lanterne magique décrit dans le Cahier 6. Le texte publié, qui a substitué les retours de promenades à Combray à celles effectuées dans les environs de Réveillon, reprend les éléments de cette évocation : ainsi, « la petite forêt triangulaire » (I, p. 9) dont sort Golo semble être née du « triangle irrégulier bleuâtre » formé par les moutons dans la campagne de Réveillon, tandis que la mystérieuse châtelaine qui accompagne le protagoniste condense, dans le flou des déterminations qui servent à la caractériser - « la dame du château », « ma compagne »-, les traits à la fois de Geneviève de Brabant et de la future duchesse de Guermantes, dont le lien de parenté sera dévoilé dans «Combray » (I, p. 103, 169, 173) 27 .

25. On verra plus loin que cet ordre n'est pas anodin : il peut être interprété à la lumière de la suite du récit, qui prélude au « drame du coucher ». 26. L'épisode apparaît dans le Cahier 6 (fos $2 \mathrm{r}^{\mathrm{o}}, 6-7 \mathrm{ros}, 5$ vo), puis est développé dans une addition aux fos $9-11$ vos du Cahier 8 où il vient se greffer sur le thème des chambres ; ce n'est qu'au moment où il corrige la première dactylographie du «Temps perdu » que Proust retranche l'épisode de la lanterne magique de l'ouverture du récit pour le placer plus loin, en prélude au drame du coucher : voir P.-L. Rey et J. Yoshida, $C S$, « Notice », I, p. 1064.

27. Notons que tout ce fragment du Cahier 1 a été directement intégré aux pages destinées au dernier volume du cycle d'Albertine : dans le texte publié d'Albertine disparue, c'est Gilberte qui incarne la châtelaine qui accompagne le héros lors de promenades nocturnes autour de Tansonville (IV, p. 267). 


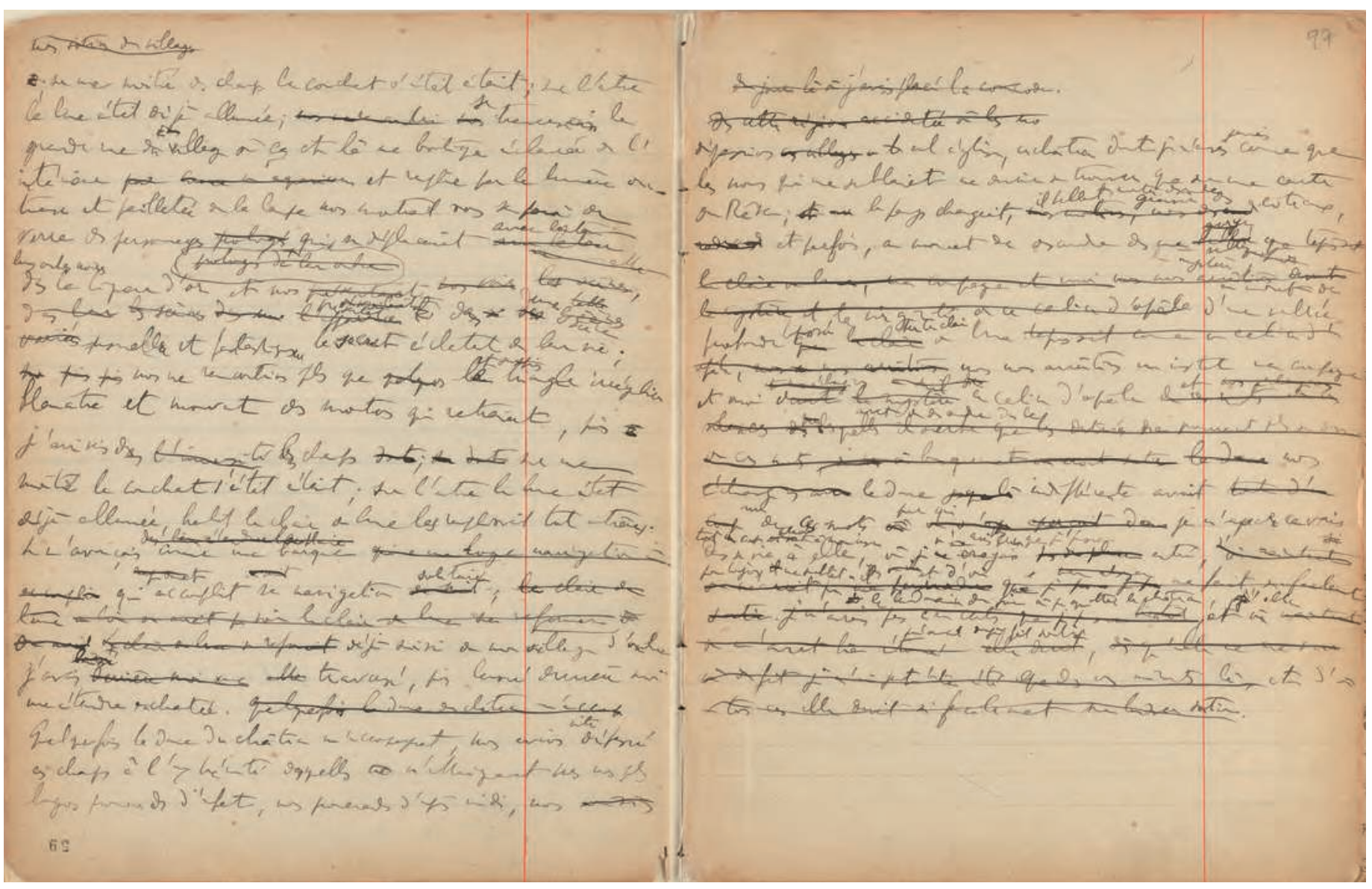

Fig. 5 : Cahier 1, fos 59 ro-58 vo

(BnF, NAF 16641)

En amont, le premier micro-récit de l'incipit semble condenser, dans un scénario minimal et schématique centré sur la figure du voyageur rejoignant de nuit une station de chemin de fer en rase campagne, les éléments épars de ces épisodes successivement travaillés dans les cahiers, offrant ainsi une superposition fulgurante de Golo dans sa chevauchée nocturne et du protagoniste au retour de ses promenades tardives. En effet, «l'étendue de la campagne déserte », " par une de ces belles nuits pavées de clair de lune » (Cahier 1, fos 71-70 vos), fait écho à la fois au décor féerique dans lequel se meuvent Geneviève et Golo (une 《lande » tour à tour « dorée » dans le Cahier 6 et « jaune » dans le Cahier 8) et à l'« étendue enchantée » baignée de clair de lune aux alentours de Réveillon. Ce sont les mêmes éléments encore que l'on trouve dans les fragments des Cahiers 6 et 8 évoquant la lecture de George Sand par la mère du héros, le fameux soir du « drame du coucher » où le père consentit à ce qu'elle passât la nuit auprès de son fils : « Mais après que Maman se fut couchée et endormie, la nuit magique du Champi sous la forêt enchantée par le clair de lune $[\ldots] »($ Cahier 8, fo 44 ro).

Dans le texte publié, ces éléments sont absents du «drame du coucher », mais le lien avec les deux premiers micro-récits de l'incipit y est explicitement marqué par la comparaison entre la chambre de Combray, rendue encore plus étrangère à l'enfant par l'éclairage de la lanterne magique, et « une chambre d'hôtel ou de "chalet", où [il fût] arrivé pour la première fois en descendant de chemin de fer ${ }^{28} »(I$, p. 9). En outre, la version définitive du premier

28. Dans les versions antérieures, le lien avec les micro-récits de l'incipit n'était pas si explicite : dans le Cahier 8, «le changement d'éclairage causé par la lanterne » donne au protagoniste l'impression d'être dans un hôtel inconnu en bord de mer (fo 11 vo). 
micro-récit condense deux éléments essentiels au prélude du « supplice du coucher » : ainsi des « adieux sous la lampe étrangère » qui suivent le voyageur « dans le silence de la nuit », en un écho discret à l'enfant quittant la salle à manger éclairée pour monter seul affronter l'obscurité de sa chambre à coucher ; enfin, la mention du « chant d'un oiseau dans une forêt » (I, p. 4), absente des premières rédactions du passage, pourrait bien être un écho à la forêt de Golo en même temps qu'un résidu de celle du Champi. Ainsi pourrait-on lire sous ce premier micro-récit, qui relate un scénario imaginaire en apparence euphorique - « excitation », « gaîté » « plaisir » dit la version du Cahier $1-$, un texte enfoui où se dessine sous les traits réjouis du voyageur se hâtant de rejoindre la prochaine gare pour rentrer chez lui, la figure angoissée de l'enfant qui, les soirs où Swann vient dîner ou bien les soirs de retour de promenade du côté de Guermantes, monte se coucher seul, privé du baiser maternel, obligé de traverser l'obscurité de la nuit « sans viatique 29 » (I, p. 27).

On voit ainsi se dessiner des liens qui établissent une cohérence discrète, mais essentielle, entre les « capsules » narratives de l'incipit et les éléments qui, au sein du récit du «drame du coucher », contribuent à dramatiser l'épisode. Ainsi, le personnage de Golo "plein d'un affreux dessein ", tel qu'il est introduit dans le Cahier 8 (fo 9 vo), offre la première incarnation de la cruauté dans le roman, en prélude à la première scène de sadisme : le « supplice » infligé à la grand-mère par la grand-tante du héros qui incite le grand-père à boire du cognac dans le jardin de Combray, juste avant la scène du coucher. Les épisodes de la lanterne magique et du « supplice du cognac » trouvent ainsi leur cohérence au sein du texte définitif autour non seulement de la notion de supplice, mais aussi du personnage de la grand-tante qui fait le lien entre les deux scènes et endosse à son tour le rôle de persécutrice ou du moins de complice ${ }^{30}$ : c'est elle, déjà, qui accompagne de sa lecture la chevauchée criminelle de Golo. Le sentiment de culpabilité éprouvé par le héros, qui se juge impuissant à éviter le supplice, est mis en évidence à la fin des deux scènes, la perversité de Golo et de la grandtante provoquant l'une un examen de conscience, l'autre le refuge dans le plaisir solitaire : la scène de masturbation dans « une petite pièce sentant l'iris » (I, p. 12), qui reprend la scène de la masturbation juvénile introduite dans le Cahier 5 (fo 109 vo) puis retravaillée dans le Cahier 1 (fo $68 \mathrm{vo}$ ) en relation avec le souvenir des boucles, est donnée en contrepoint de la scène de supplice du cognac comme si elle en était la conséquence directe.

C'est peut-être la même logique sous-jacente qui, dans l'incipit, explique que le supplice des boucles tirées - il s'agit visiblement d'un châtiment, impliquant en amont une faute du protagoniste - suscite immédiatement la rêverie érotique autour de « la fille de mon rêve » (I, p. 5). Ainsi, ce lien implicite entre supplice, culpabilité et sensualité, pourrait avoir motivé le déplacement du souvenir des boucles tirées juste avant la rêverie érotique à l'ouverture de la Recherche, contrairement à l'ordre donné dans le Cahier 1, où la rêverie érotique précédait le souvenir des boucles (fos 70-69 vos).

Cette relecture génétique des premières pages de l'œuvre aura permis de dégager la cohérence des quatre départs fictionnels donnés par le « dormeur éveillé » autour d'un motif insistant, le supplice, dont la violence, quasi occultée en amont de la scène du coucher dans le texte publié en 1913, est évidente dans les avant-textes dont les réécritures successives ont permis la mise en place de l'incipit de la Recherche. Les scénarios minimalistes ébauchés à l'ouverture de Du côté de chez Swann portent la trace de motifs progressivement gommés : les éléments

29. La comparaison entre les figures de l'enfant et du voyageur était plus insistante encore dans Jean Santeuil, où l'angoisse de Jean au moment de se coucher était décrite comme « l'horrible souffrance indéfinissable qui peu à peu devenait grande comme la solitude, comme le silence et comme la nuit », dans laquelle il ne pouvait s'engloutir que muni du baiser maternel, véritable « viatique » offert « pour qu'il accomplît sans terreur le voyage souterrain, traversât rassasié les royaumes sombres » (JS, p. 205). Notons qu'à l'approche de l'heure fatidique du coucher, cette angoisse se trouvait un instant conjurée par la «grosse lampe », « épanouissant sa lumière cordiale » (ibid.) ; ce chapitre consacré au coucher de Jean se clôt par un développement plus général sur l'habitude qui, seule, avait pu le guérir de son angoisse d'enfant, mais dont l'effet ne pouvait rien contre l'inquiétude qu'il devait éprouver toute sa vie de coucher « dans une chambre inconnue, dans un château ou à l'hôtel » (ibid., p. 209).

30. Le texte du Côté de Guermantes confond d'ailleurs, dans un rappel de la scène du cognac, la grand-tante et le grand-oncle (II, p. 471), preuve que cette dernière peut à bon droit figurer dans la liste des «persécuteurs » aux côtés de ses complices masculins. 
renvoyant au thème de la séparation avec la mère ont ainsi été distribués dans d'autres épisodes du roman, en lien avec le drame du coucher vers lequel convergent tous les fils effacés. Hors de toute enquête génétique, c'est la perception par le (re)lecteur de l'œuvre de cette cohérence profonde des micro-scénarios de l'incipit, en lien avec d'autres moments et d'autres lieux du roman, qui lui fait ressentir, comme l'a bien montré Michel Charles, que ce début est « gros » de virtualités narratives : malgré - il faudrait plutôt dire à cause de - leur caractère allusif, la matière romanesque de la Recherche semble sortir (presque) tout entière de ces premières ébauches de récit, comme Combray de la tasse de thé du héros.

Il me semble que le début de la Recherche gagne une nouvelle lisibilité à la lumière de ce « croisement » entre analyse génétique et théorie des textes possibles : on peut en effet y lire la mise en scène du processus qui a permis qu'émerge la Recherche à partir du projet du Contre Sainte-Beuve, et avant lui, de ces éparses « Pages écrites »dont l'énumération à la fois récapitulative et programmatique dans le Carnet 1 ne pouvait suffire à construire une cohérence. À l'incipit du roman tel qu'il prend forme à la charnière des années 1908-1909, se joue la mise en route de la "machine fictionnelle », dont les micro-récits analysés apparaissent comme les premiers ronronnements : aussitôt ébauchée, chaque "capsule » fictionnelle se referme pour laisser place à la suivante, de sorte que chacune semble fonctionner de manière quasi autonome, en marge du destin du « dormeur éveillé ». Ces amorces narratives anticipent le premier départ de la fiction avec la remémoration volontaire du « drame du coucher », qui constituera à son tour un « faux départ », avant la restitution complète du souvenir déclenchée par l'épisode de la madeleine. L'enjeu de ces premières pages est bien d'instituer l'instance d'énonciation à la fois en tant que personnage de fiction et en tant que sujet producteur de cette fiction, et ce par le truchement d'une série d'ébauches de récits qui esquissent, sur le mode de la «variante par anticipation », comme dirait un théoricien des textes possibles, le drame du coucher. Ainsi, le début de la Recherche mime à sa manière la genèse de la fiction en exhibant les rouages d'une énonciation productrice de fables, contribuant ainsi à définir la forme romanesque comme l'espace du déploiement des possibles. 
MAYa LAVAult, professeur agrégée de Lettres modernes, chercheuse rattachée à l'équipe Proust de l'ITEM (CNRS-ENS), a soutenu en décembre 2009 une thèse de doctorat intitulée « Des secrets à l'œuvre : formes et enjeux romanesques du secret dans À la recherche du temps perdu de Marcel Proust », qui sera prochainement publiée aux éditions Champion. Elle est l'auteur de plusieurs articles autour des motifs du secret dans la Recherche et de la conception proustienne de la fiction comme exploration des possibles.

mlavault@gmail.com

\section{Du côté de l'incipit de la Recherche : la genèse de la fiction selon Proust}

Cette analyse des premières pages de la Recherche vise à combiner approche génétique et théorie des textes possibles pour montrer que les micro-récits qui ont progressivement pris place à l'ouverture de l'œuvre dessinent une cohérence dont la lisibilité s'est trouvée effacée au fil du processus de genèse, mais dont le lecteur du texte publié en 1913 peut percevoir des traces qui continuent de hanter ce début de roman. En effet, les réécritures successives de l'incipit, depuis les cahiers de 1908-1909, ont contribué à effacer les motifs du supplice et de la séparation avec la mère, qui relient entre eux les micro-récits formés par le « dormeur éveillé », pour les déplacer, dans le texte définitif, au sein de l'épisode du « drame du coucher ». Se joue ainsi dans ces premières pages la mise en route de la fiction, dont la genèse est en quelque sorte mimée par la succession de ces ébauches de récits fictionnels qui introduisent le lecteur dans un espace ouvert à l'exploration des possibles narratifs.

This study of the first pages of $\grave{A}$ la recherche du temps perdu aims at combining the genetic approach and the "possible texts" theory (inspired by Michel Charles, before many present day critics) in order to point out that the short narratives which gradually took place at the beginning of the novel have a global frame: although this obvious frame was erased along the genetic process, the reader of the text published in 1913 can notice its marks still haunting these first pages. As a matter of fact, the successive rewritings, from the 1908-1909 manuscripts, arased the pattern of the dire separation from the mother: in the published version, this pattern, which used to unify the few short narratives that the "awakened sleeper" shaped at the beginning of the novel, was moved further on to the bedtime scene. Thus the reader of the beginning of $\grave{A}$ la recherche du temps perdu attends the starting of the fictional process: it seems that the sequence of short narratives outlined in the first pages enacts the very genesis of Proust's fictional manner, as an exploration of narrative potentialities.

Diese Untersuchung der ersten Seiten von Auf der Suche nach der verlorenen Zeit hat zum Ziel, einen genetischen Ansatz mit der Theorie der ,möglichen Texte“ zu verbinden, um so aufzuzeigen, dass die zu Beginn des Werks allmählich auftauchenden kurzen Erzählungen in einem größeren Zusammenhang stehen. Obwohl dieser im Verlauf des Entstehungsprozesses verwischt wurde, kann der Leser des 1913 veröffentlichten Textes jedoch Spuren davon erkennen, welche noch immer auf diese ersten Seiten des Romans verweisen. Tatsächlich haben die sukzessiven Überarbeitungen des Incipit seit den Heften von 1908-1909 die Motive der Qual und der Trennung von der Mutter, welche die vom ,erwachten Schläfer“ geschaffenen kurzen Erzählungen verbinden, ausgelöscht, um sie im endgültigen Text im ,drame du coucher" einzubauen. Diese ersten Seiten von Auf der Suche nach der verlorenen Zeit bedeuten ebenso den Beginn des Prozesses der Fiktion, dessen Entstehung in gewisser Weise von dieser Reihe von Entwürfen fiktiver Erzählungen als Erforschung möglicher Narrative geprägt zu sein scheint.
Este análisis de las primeras páginas de En busca del tiempo perdido trata de combinar el enfoque genético y la teoría de los textos posibles para mostrar que los microrrelatos que han ido ocupando los inicios de la obra establecen una coherencia, cuya legibilidad se ha desdibujado durante el proceso de génesis, pero que un lector del texto publicado en 1913 puede todavía percibir a través de las huellas que siguen habitando ese comienzo de la novela. En efecto, las reescrituras sucesivas del incipit, a partir de los cuadernos de 1908-1909, contribuyeron a borrar los motivos del suplicio y de la separación de la madre, que vinculan los microrrelatos formados por el "durmiente despierto", para desplazarlos, en el texto definitivo, dentro del episodio del "drama de acostarse”. Queda representada así, en esas primeras páginas, la puesta en marcha de la ficción, cuya génesis aparece, de alguna manera, desdoblada en la sucesión de esos esbozos de relatos ficticios, que introducen al lector en un espacio abierto a la exploración de los posibles narrativos.

L'analisi qui condotta intorno alla zona incipitaria della Recherche combina l'approccio genetico con i criteri propri della teoria della creazione letteraria per mostrare come i microsegmenti narrativi che hanno progressivamente trovato la loro collocazione in quella zona d'apertura rivelino una coerenza la cui la leggibilità si è andata cancellando lungo la strada del processo genetico, ma di cui lettore del testo pubblicato nel 1913 è in grado di rintracciare i segni che continuano a inquietare l'avvio del romanzo. Infatti, le successive riscritture dell'incipit, a valle dei quaderni del 1908-1909, hanno contribuito a rimuovere i motivi di quel tormento e della separazione dalla madre, collegando fra loro i microracconti formati dal "dormeur éveillé", per spostarsi, nella stesura definitiva, entro l'episodio del "drame du coucher". Così, il lettore assiste sin dalle prime pagine all'avviarsi del processo di finzione, la cui genesi è, in un certo senso, mimata dalla sequenza di brevi racconti che introducono il lettore in uno spazio aperto all'esplorazione di possibili sviluppi narrativi.

Esta análise das primeiras páginas da Recherche pretende combinar uma abordagem genética com a teoria dos textos possíveis para mostrar como as micro-narrativas que ocorrem gradualmente no início da obra possuem uma coerência nem sempre evidente durante o processo de génese, mas de que o leitor do texto publicado em 1913 pode detectar os vestígios. De facto, sucessivas reescritas do incipit, após os cadernos de 1908-1909, tiveram o efeito de esbater os motivos do suplício e da separação da mãe, que conectavam entre si as micro-narrativas formadas pelo "adormecido desperto" e as deslocaram, no texto definitivo, para o episódio do "drama do deitar". Assim se põe em marcha logo nas primeiras páginas a ficção, cuja génese é de certa forma imitada pela sucessão desses esboços de histórias fictícias que trazem o leitor para um espaço aberto à exploração das possibilidades narrativas. 